OPEN ACCESS

Edited by:

Tom Carr,

Michigan State University, USA

Reviewed by:

Christopher I. Petkov,

Newcastle University, UK

Herbert Heuer,

Leibniz Research Centre for Working

Environment and Human Factors,

Germany

*Correspondence:

Christopher M. Conway

cconway@gsu.edu

Specialty section:

This article was submitted to

Cognition,

a section of the journal

Frontiers in Psychology

Received: 08 October 2015 Accepted: 08 February 2016

Published: 25 February 2016

Citation:

Walk AM and Conway CM (2016) Cross-Domain Statistical-Sequential

Dependencies Are Difficult to Learn.

Front. Psychol. 7:250.

doi: 10.3389/fpsyg.2016.00250

\section{Cross-Domain Statistical-Sequential Dependencies Are Difficult to Learn}

\author{
Anne M. Walk ${ }^{1}$ and Christopher M. Conway ${ }^{2 *}$ \\ ${ }^{1}$ Neurocognitive Kinesiology Lab, Department of Kinesiology and Community Health, University of Illinois at \\ Urbana-Champaign, Urbana, IL, USA, ${ }^{2}$ NeuroLearn Lab, Department of Psychology, Georgia State University, Atlanta, GA, \\ USA
}

Recent studies have demonstrated participants' ability to learn cross-modal associations during statistical learning tasks. However, these studies are all similar in that the cross-modal associations to be learned occur simultaneously, rather than sequentially. In addition, the majority of these studies focused on learning across sensory modalities but not across perceptual categories. To test both cross-modal and crosscategorical learning of sequential dependencies, we used an artificial grammar learning task consisting of a serial stream of auditory and/or visual stimuli containing both within- and cross-domain dependencies. Experiment 1 examined within-modal and cross-modal learning across two sensory modalities (audition and vision). Experiment 2 investigated within-categorical and cross-categorical learning across two perceptual categories within the same sensory modality (e.g., shape and color; tones and nonwords). Our results indicated that individuals demonstrated learning of the within-modal and within-categorical but not the cross-modal or cross-categorical dependencies. These results stand in contrast to the previous demonstrations of cross-modal statistical learning, and highlight the presence of modality constraints that limit the effectiveness of learning in a multimodal environment.

Keywords: statistical learning, implicit learning, sequential learning, cross-modal learning, multisensory integration, modality constraints, artificial grammar learning

\section{INTRODUCTION}

Many organisms have the ability to detect invariant patterns and associations from a seemingly chaotic environment. One such ability, statistical-sequential learning, involves the learning of statistical patterns across items presented in sequence (Saffran et al., 1996; Daltrozzo and Conway, 2014). Statistical learning appears to be central to the development of many cognitive functions, especially language (Saffran et al., 1996; Conway et al., 2010; Misyak et al., 2010; Nemeth et al., 2011; Arciuli and Simpson, 2012; Kidd, 2012; Misyak and Christiansen, 2012). Traditionally, statistical learning has been studied in a unimodal manner, presenting participants with stimuli to a single sensory modality, such as audition, vision, or touch (Saffran et al., 1996; Fiser and Aslin, 2001; Kirkham et al., 2002; Conway and Christiansen, 2005). However, in many natural circumstances, such as spoken language, multiple sensory modalities are involved. For example, sighted individuals make extensive use of visual facial information, such as the movement of the mouth, to aid in speech perception (Rosenblum, 2008).

Despite the importance of multisensory integration in language processing and other areas of cognition, only recently has multisensory integration been investigated in the context of statistical 
learning. Toward this end, Mitchel and Weiss (2011) presented unimodal auditory and visual input streams simultaneously to participants and manipulated the audiovisual correspondence across the two modalities. They found that learners could extract the statistical associations in both input streams independently of the other (consistent with the findings of Seitz et al., 2007) except when the triplet boundaries were desynchronized across the visual and auditory streams. In such conditions, learning was disrupted, suggesting that statistical learning is affected by cross-modal contingencies. Other studies have similarly shown that input presented in one modality can affect learning in a second concurrently presented modality. For instance, Cunillera et al. (2010) showed that simultaneous visual information could improve auditory statistical learning if the visual cues were presented near transition boundaries (see also Robinson and Sloutsky, 2007; Sell and Kaschak, 2009; Mitchel and Weiss, 2010; Thiessen, 2010). More recently, Mitchel et al. (2014) used the McGurk illusion to demonstrate that learners can integrate auditory and visual input during a statistical learning task, suggesting that statistical computations can be performed on an integrated multimodal representation.

Although these studies are all clear demonstrations of multimodal integration during statistical learning tasks, they use concurrent auditory and visual input. That is, the visual and auditory inputs were presented simultaneously, and learners were tested on their ability to learn these simultaneous crossmodal associations. No studies to our knowledge have tested the extent that cross-modal statistical associations can be learned and integrated across time as elements in a sequence, in which an auditory stimulus (e.g., a tone) might be associated with the next occurrence of a particular visual stimulus (e.g., a shape) or vice-versa. In addition, previous studies have used multi-sensory patterns containing cross-modal regularities across sensory modalities, but none to our knowledge have tested learning of dependencies across different perceptual categories but that are within the same sensory modality (i.e., color and shape or tones and non-words). It is possible that learning cross-modal dependencies may have different computational demands than the learning of cross-categorical dependencies, perhaps due to differences in perceptual or attentional requirements.

The aim of the present study, therefore, was to investigate the limits of cross-domain statistical-sequential learning. From a purely associative learning framework, it might be hypothesized that statistical patterns should be learned just as readily between stimuli regardless of their modality or perceptual characteristics (i.e., learning a dependency between items A and B should not be any different than learning a dependency between items A and C). Such an unconstrained view of statistical learning was common in its early formulations (see Frensch and Runger, 2003 and Conway et al., 2007 for discussion). However, it is now known that statistical learning is constrained by attentional and perceptual factors. For example, statistical learning of non-adjacent relationships is heavily influenced by perceptual similarity, with learning improving when the nonadjacent elements are perceptually similar to one another (i.e., have a similar pitch range or share some other perceptual cue;
Creel et al., 2004; Newport and Aslin, 2004; Gebhart et al., 2009). Likewise, Conway and Christiansen (2006) proposed that statistical learning is analogous to perceptual priming, in which networks of neurons in modality-specific brain regions show decreased activity when processing other items within the same modality that have similar underlying regularities or structure (see also, Reber et al., 1998; Chang and Knowlton, 2004; Conway et al., 2007). Recent neuroimaging evidence confirms that statistical learning is mediated at least in part by processing in unimodal, modality-specific brain regions (Turk-Browne et al., 2009) - in addition to involving "downstream" brain regions that appear less tied to a specific perceptual modality including Broca's area, the basal ganglia, and the hippocampus (Lieberman et al., 2004; Opitz and Friederici, 2004; Petersson et al., 2004; Abla and Okanoya, 2008; Karuza et al., 2013; Schapiro et al., 2014). Thus the existing literature suggests that statistical learning involves a combination of bottom-up perceptual processing via unimodal, modality-specific mechanisms, but also more domain-general learning and integration processes that perhaps occur further downstream (Keele et al., 2003; Conway and Pisoni, 2008; Daltrozzo and Conway, 2014; Frost et al., 2015).

Thus, the learning of sequential patterns appears to be at least partly constrained by the nature of the sensory and perceptual processes that are engaged. Another way to think of this is that statistical learning is likely influenced by Gestalt-like principles that make it easier to learn associations between items in the same modality or that share perceptual features (Newport and Aslin, 2004). Consequently, statistical learning of cross-modal or crosscategorical sequential associations might be more challenging than the previous empirical research seems to indicate. It is possible that the previous demonstrations of multisensory integration during statistical learning tasks that used concurrent auditory and visual input (e.g., Cunillera et al., 2010; Mitchel and Weiss, 2011; Mitchel et al., 2014) were less cognitively demanding than learning elements across a temporal sequence. It is currently an open question to what extent statisticalsequential cross-modal and cross-categorical dependencies can be learned.

To test cross-modal and cross-categorical statistical learning, we employed an artificial grammar learning (AGL) paradigm, commonly used to study implicit and statistical learning (Seger, 1994; Perruchet and Pacton, 2006), in which stimuli are determined by a finite state grammar. Unlike previous statistical learning or AGL tasks, our paradigm used a series of inputs from different sensory modalities and/or perceptual categories, with each individual unit presented in succession. In this manner, we could test whether participants can learn cross-domain dependencies across the temporal sequence. The grammar itself (see Figure 1), created by Jamieson and Mewhort (2005) and also used by Conway et al. (2010), has certain advantages over other artificial grammars commonly used. First, unlike most other grammars including the classic "Reber" grammar (Reber, 1967) and countless others, there are no positional constraints. That is, each element of the grammar can occur at any position, with equal frequency, preventing position information - such as which elements or pairs of elements occur at the beginning 


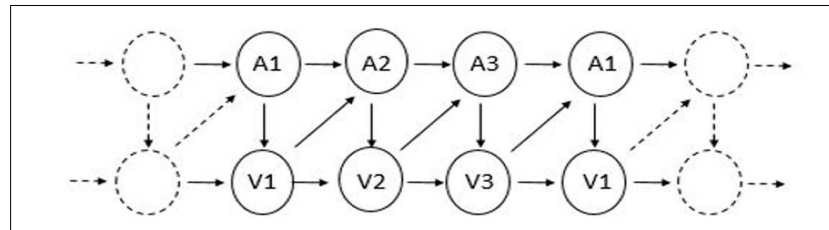

FIGURE 1 | The artificial grammar used in both Experiments. "V" and "A" refer to visual and auditory stimuli, respectively.

versus the ending of sequences - from becoming a confound. Second, there are also no constraints on sequence length. A large set of stimuli can be generated at a particular length (such as length 6 used in the present study), preventing sequence length from becoming a confound. Finally, the grammar describes the probability in which a successive element $(n+1)$ can occur given the previous element $(n)$. This means that primarily firstorder element transitions are contained in the grammar; thus, "learning the grammar" in this case generally means one thing: learning the forward-transition, adjacent element statistics ${ }^{1}$, making interpretation about what is learned or not learned relatively straightforward. Consequently, this also makes it easy to design sequences containing both cross-domain and withindomain dependencies.

In Experiment 1, participants were exposed to input sequences generated from the artificial grammar that were composed of tones interspersed with pictures of shapes. Importantly, the sequences consisted of both within-modal (e.g., tone-tone or shape-shape) and cross-modal associations (e.g., tone-shape or shape-tone). In Experiment 2 the sequences were composed of stimuli from two different perceptual categories within the same sensory modality (shapes and colors for the visual stimuli and tones and single syllable non-words for the auditory stimuli), allowing us to test cross-categorical learning. By incorporating a combination of within- and cross-modality stimuli (Experiment 1) and within- and cross-category stimuli (Experiment 2), we were able to examine to what extent participants naturally learn statistical-sequential patterns across sensory domains and perceptual categories.

\section{EXPERIMENT 1: LEARNING ACROSS SENSORY MODALITIES}

\section{Materials and Methods \\ Participants}

Fifteen undergraduate students from a Midwest university participated (Age Range $=18-23$; Mean Age = 18.93; Females $=9$ ). All were fluent English speakers. All participants

\footnotetext{
${ }^{1}$ As pointed out by one of the reviewers of the current manuscript, non-adjacent dependencies are also contained in the grammar that could possibly be learned by participants. Each element in the grammar has two within-domain nonadjacent dependencies and one cross-domain non-adjacent dependency, with each following with equal probability ( 0.33 each). For example, A1 can be followed by A3 (with an intervening A2), V2 (with an intervening V1 or A2), or A2 (with an intervening V1). To what extent these non-adjacent dependencies impact performance will be discussed in more detail in the Section "Discussion."
}

were enrolled in college at the time of their participation. Participants received credit toward partial fulfillment of an undergraduate course as compensation for their time. The study was carried out in accordance with the recommendations of the Saint Louis University Institutional Review Board. All participants gave written informed consent in accordance with the Declaration of Helsinki.

\section{Stimulus Materials}

We used an artificial grammar consisting of three visual and three auditory elements. The visual elements were abstract black and white shapes used previously in a study by Joseph et al. (2005) and considered difficult to verbally label. The auditory elements were three pure tones that were generated using Audacity software, having frequencies of 210,286 , and $389 \mathrm{~Hz}$, which neither conform to standard musical notes nor have standard musical intervals between them (as used in Conway and Christiansen, 2005).

Each sequence was generated by an artificial grammar with constrained probabilities (similar to those used in Jamieson and Mewhort, 2005 and Conway et al., 2010; See Figure 1). The grammar dictates that any given element can be followed by one element from the same sensory modality and one element from the other sensory modality. For example, if $\mathrm{V} 1$ is the starting element, it can be followed by either A2 or V2 with an equal probability $(50 / 50 \%)$. Thus, V1-A2-A3-V3-A1-V1 is an example of a sequence that could be generated by this grammar; it contains four cross-modal dependencies (V1-A2; A3-V3;

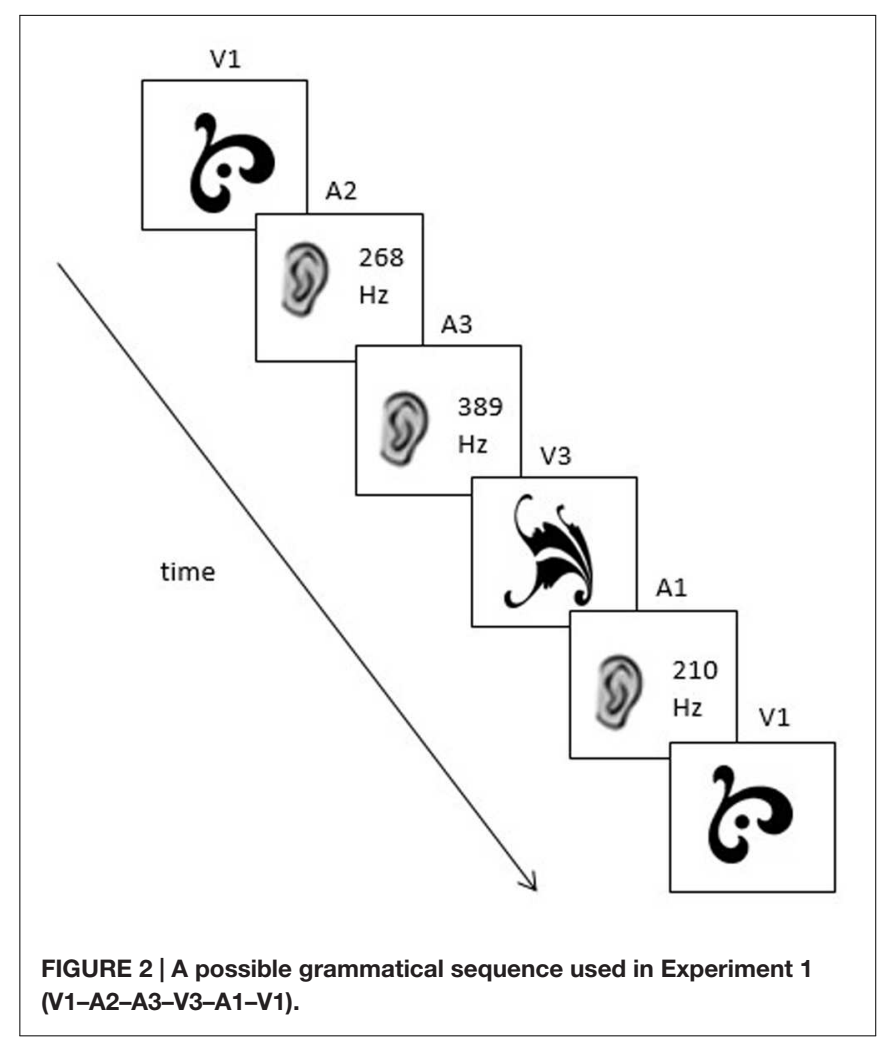


V3-A1; A1-V1) and one within-modal dependency (A2-A3; see Figure 2).

Using the grammar presented in Figure 1, a single "learning" stream was generated and used for all participants, consisting of 180 stimuli presented in sequence. In addition, three types of six-item test sequences were constructed: grammatical sequences, ungrammatical sequences containing within-modal violations, and ungrammatical sequences containing cross-modal violations. To create within-modal violation sequences, all within-modal dependencies were altered so that they violated the grammar, with the cross-modal dependencies remaining grammatical. For cross-modal violation sequences, all cross-modal dependences were altered so that they violated the grammar, with the withinmodal dependencies remaining grammatical. For example, in the case of a within-modal violation sequence, if the grammatical sequence was $\mathrm{V} 1-\mathrm{A} 2-\mathrm{A} 3$, the element $\mathrm{A} 3$ would be replaced with the other auditory element, so that the sequence would become V1-A2-A1. From that point, the grammar would be renewed and would continue correctly until another within-modal transition occurred. We constructed 20 grammatical test sequences, 10 within-modal ungrammatical test sequences, and 10 cross-modal ungrammatical test sequences. The total number of violations in the within-modal violation stimulus set (28 violations total or 2.8 violations on average per sequence) and cross-modal violation stimulus set (25 violations total or 2.5 violations on average per sequence) were roughly equal and not statistically different from each other $(t=0.669, p=0.512)$. All test sequences are listed in the Appendix (Table A1).

\section{Procedure}

All participants completed a learning phase and a test phase. In the learning phase, participants were seated in front of a computer monitor with a pair of headphones. They were instructed to pay attention to the pictures and sounds that were displayed. Participants were exposed to the continuous stream of 180 shapes and tones that was generated using the grammar. The durations for both the auditory and visual stimuli were $1000 \mathrm{~ms}$ each, with an ISI of $1000 \mathrm{~ms}$, giving a total learning phase duration of $6 \mathrm{~min}$.

In the test phase of the experiment, participants were told that the input stream they had observed was created according to certain rules that determined the order that each element was presented. Participants were then presented with each of the six-item test sequences and were asked to determine if each item "followed the rules" (i.e., was grammatical) or "did not follow the rules" (i.e., was ungrammatical). Participants responded by pressing one of two buttons to indicate their choice. Participants were exposed to the novel grammatical, within-modal ungrammatical, and cross-modal ungrammatical sequences in random order. Within each test sequence, the stimulus durations $(1000 \mathrm{~ms})$ and ISI $(1000 \mathrm{~ms})$ were the same as used in the learning phase. Participants had as much time as needed to make their response, after which the next test trial began. Note that for both the learning and test phases the auditory and visual tokens were randomly assigned and mapped to the elements of the grammar. For one participant A1 might be the $210 \mathrm{~Hz}$ tone, but for another participant A1 might be the $286 \mathrm{~Hz}$ tone, etc. Thus, even though each participant received the same learning and test items in terms of their underlying structural patterns, the actual tokens that mapped onto these patterns differed for each participant, determined randomly.

\section{Results and Discussion}

Results are shown in Table 1, displaying the percentage of test items classified correctly for each test item type. Performance on the within-modal violation sequences was numerically the highest $(M=64.00 \%)$ followed by performance on the grammatical sequences $(M=60.67 \%)$, and lastly the cross-modal violations $(M=48.67 \%)$. To explore the accuracy of participants' performance on the three item types, a repeated measures analysis of variance (ANOVA) was conducted, indicating a significant main effect of sequence type $[F(2,28)=4.893$, $\left.p=0.015, \eta_{\mathrm{p}}^{2}=0.259\right]$. A test of simple comparisons with a Bonferroni correction indicated that there was a statistically significant difference between performance on the within-modal violations and the cross-modal violations $(p<0.05)$. A series of single sample t-tests was run to test the average performance on each item type to chance (50\%). The analysis indicated that participants performed significantly above chance on the grammatical items $(t=4.00, p \leq 0.05$, Cohen's $d=1.04)$ and the within-modal ungrammatical items $(t=4.18, p \leq 0.001$, $d=1.08$ ), but not on the cross-modal ungrammatical items $(t=-0.31, p \geq 0.10, d=0.08)^{2}$

The findings from Experiment 1 indicate that participants were more proficient at detecting within-modal violations that is, violations occurring between stimuli in the same sensory modality - than at detecting cross-modal violations that occurred between stimuli in different modalities. In fact, participants were completely unable to successfully detect violations of cross-modal contingencies. The lack of cross-modal learning stands in contrast to previous studies of multimodal statistical learning, which differed from the present study by

\begin{abstract}
${ }^{2}$ Due to a design error, two within-modal violation test items (\#25 and \#26) were found to be problematic. Instead of all within-modal transitions being ungrammatical, each had one within-modal dependency that was not violated. In addition, these two items began with a repetition of a single stimulus, which could add unwanted salience to those items. Thus, all statistical analyses were rerun after taking out participant responses to these two items. The overall pattern of results remained similar: participants performed statistically better than chance on the grammatical items $(M=60.67 \%, t=4.00, p=0.001, d=1.03)$ and the withinmodal violation items $(M=60.00 \%, t=2.70, p=0.017, d=0.70)$ but not on the cross-modal violation items $(M=48.67 \%, t=-0.31, p=0.764)$.
\end{abstract}

TABLE 1 | Mean Performance (percent correct) and standard deviations for Experiments 1 and 2.

\begin{tabular}{|c|c|c|c|}
\hline & \multirow{2}{*}{$\begin{array}{l}\text { Experiment } 1 \\
\text { (Within- vs. } \\
\text { cross-modal) }\end{array}$} & \multicolumn{2}{|c|}{$\begin{array}{c}\text { Experiment } 2 \\
\text { (Within- vs. cross-category) }\end{array}$} \\
\hline & & Visual & Auditory \\
\hline Grammatical & 60.67 (16.85) & $51.88(17.11)$ & $60.63(11.81)$ \\
\hline Within-modal/category & $64.00(12.98)$ & $65.00(18.97)$ & 80.63 (15.69) \\
\hline Cross-modal/category & $48.67(10.33)$ & $51.25(21.25)$ & $50.63(19.14)$ \\
\hline
\end{tabular}


their use of simultaneous rather than sequential cross-modal dependencies.

\section{EXPERIMENT 2: LEARNING ACROSS PERCEPTUAL CATEGORIES WITHIN A SINGLE SENSORY MODALITY}

The results of Experiment 1 demonstrate that when exposed to multimodal sequential patterns, within-modal but not crossmodal violations can be detected. In Experiment 1, the crossmodal associations were multisensory (i.e., consisting of audiovisual or visual-audio links). Another way to probe multimodal learning is to test the ability to learn associations that are within the same sensory modality (e.g., vision or audition) but that exist between different perceptual categories (e.g., tones and words or colors and shapes).

\section{Materials and Methods \\ Participants}

A new group of 32 undergraduate students from the same Midwestern University participated in the study (Age Range $=18-22$; Mean Age $=19.54,1$ not reported; Females $=20$, 2 not reported). All students were fluent speakers of English. All participants were enrolled in college at the time of their participation and received credit toward partial fulfillment of an undergraduate course as compensation for their time. Participants were randomly assigned to one of two conditions, auditory or visual. The study was carried out in accordance with the recommendations of the Saint Louis University Institutional Review Board. All participants gave written informed consent in accordance with the Declaration of Helsinki.

\section{Stimulus Materials}

For Experiment 2, four types of stimuli were used: the same black and white shapes and same pure tones as before, as well as three colored circles (red, green, and blue) and three single-syllable non-words ("dak," "pel," and "vot"). This provided us with four sets of perceptual categories, two sets (tones, non-words) for the auditory modality and another two sets (shapes, colors) for the visual modality.

The learning stream and test sequences were the same as used in Experiment 1, except that the inputs were altered to reflect the two new stimulus sets. In the visual condition, $\mathrm{A}$ and $\mathrm{V}$ elements of the original grammar were replaced with shapes and colors (see Figure 3). In the auditory condition, $\mathrm{A}$ and $\mathrm{V}$ elements of the grammar were replaced with tones and non-words (see Figure 4). Mirroring the design of Experiment 1, the new stimuli formed a set of grammatical sequences, within-categorical ungrammatical sequences, and cross-categorical ungrammatical sequences.

\section{Procedure}

The procedure was the same as in Experiment 1, except that participants were assigned to one of two groups (auditory or visual). Participants assigned to the auditory group received input sequences composed of the two categories of auditory
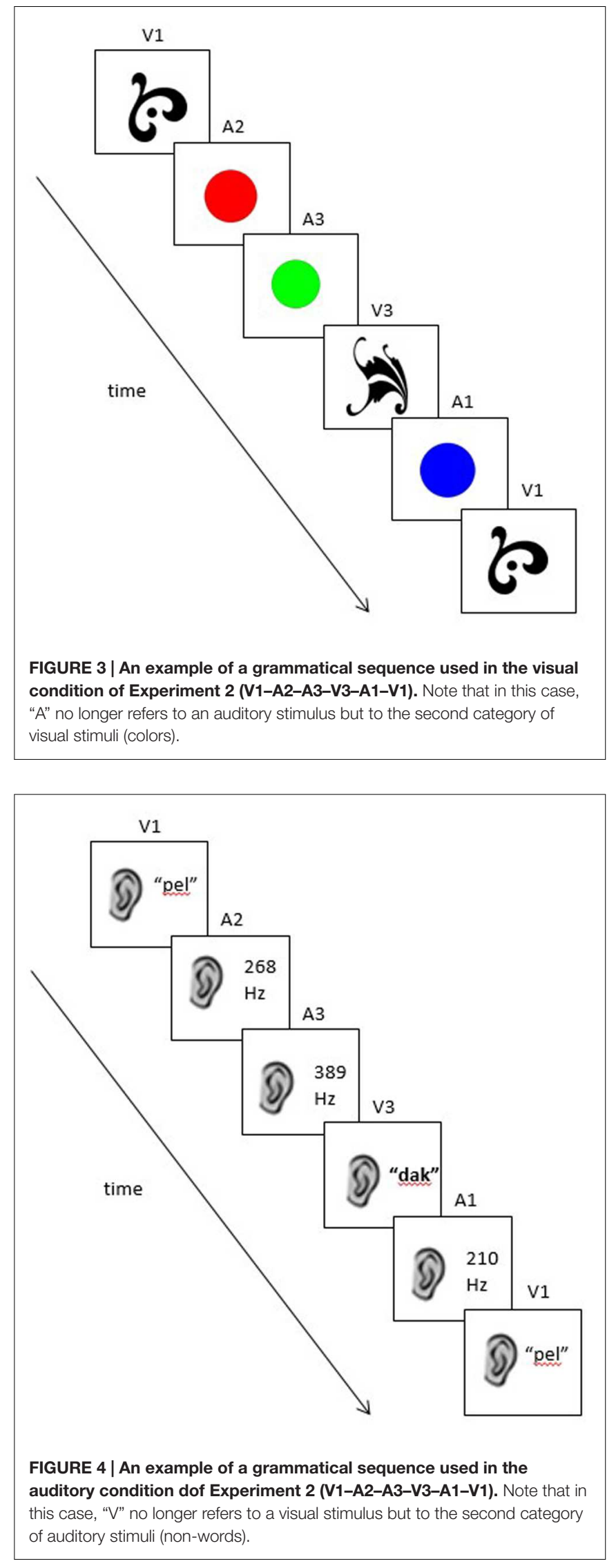
stimuli (pure tones and non-words), while participants assigned to the visual condition received sequences composed of the two categories of visual stimuli (abstract shapes and colored circles).

\section{Results and Discussion}

As in Experiment 1, the accuracy scores are displayed as percentages (Table 1). To investigate the performance of the two groups, a repeated measures, mixed factor ANOVA was conducted with group (visual and auditory) as the between subjects variable and sequence type (grammatical, within-category violations, and cross-category violations) as the within subjects variable. The results of the Mauchly's test of sphericity suggested that the sphericity assumption of a repeated measures ANOVA was violated [Mauchly's $W=0.508$. $p \leq 0.001]$. Therefore, in all subsequent analyses, the Greenhouse-Geisser test of the multivariate analysis is reported. The analysis revealed significant main effects of sequence type $\left[F(1.34,40.20)=13.56, p \leq 0.001, \eta_{\mathrm{p}}^{2}=0.311\right]$ and group $\left[F(1,30)=4.794, p \leq 0.05, \eta_{\mathrm{p}}^{2}=0.138\right]$. A test of simple comparisons with a Bonferroni correction was run to further explore the main effects. It revealed that participants across the two groups performed significantly higher on the within-category violation sequences than either the grammatical sequences ( $p \leq 0.001$ ) or the cross-category violation sequences $(p \leq 0.01)$. Across sequence type, participants in the auditory condition performed better than participants in the visual condition.

A series of independent samples $t$-tests were run on each group of participants comparing performance to chance for each sequence type. The analysis showed that participants in the visual group only had better than chance performance for the within-category items $(t=3.162, p \leq 0.01$, $d=0.79$ ). However, participants in the auditory group showed better than chance performance for both the withincategory violation items $(t=7.806, p \leq 0.001, d=1.95)$ and the grammatical items $(t=3.597, p \leq 0.01, d=0.90)$. Performance for cross-category items was at chance levels for both groups. $^{3}$

The pattern of results mirrors that seen in Experiment 1 but extends it to the learning of statistical patterns across perceptual categories within the same sensory modality. Participants were able to detect statistical-sequential violations within the same perceptual category but were unable to detect violations

\footnotetext{
${ }^{3}$ As in Experiment 1, all analyses were re-run after removing two problematic test items (\#25 and \#26) in the within-modal condition. The overall pattern of results was similar as before: the results of the ANOVA resulted in the same significant effects: Sequence type $\left[F(2,29)=13.96, p=0.000, \eta_{\mathrm{p}}^{2}=0.194\right]$ and Group $\left[F(1,30)=5.322, p=0.028, \eta_{\mathrm{p}}^{2}=0.151\right]$. In the auditory condition, participants scored significantly better than chance when responding to grammatical $(M=60.63 \%, t=3.60, p=0.003, d=0.90)$ and within-modal ungrammatical items $(M=79.69 \%, t=5.69, p=0.000, d=0.89)$, but not crossmodal ungrammatical items $(M=50.63 \%, t=0.13, p=0.90, d=0.03)$. In the visual condition, participants did not show scores significantly better than chance for any of the three sequence types: grammatical $(M=52.81 \%)$, within-modal ungrammatical $(M=59.38 \%)$, or cross-modal ungrammatical $(M=51.87 \%)$, although numerically the same overall trend is observed with highest performance on the within-modal ungrammatical items.
}

across perceptual categories. Furthermore, a modality effect was observed, consistent with previous research showing that audition displays higher levels of learning for sequentially presented patterns (Conway and Christiansen, 2005; Emberson et al., 2011).

\section{GENERAL DISCUSSION}

The findings from this study suggest that learning statisticalsequential associations within a perceptual or sensory domain is easier than learning across domains. In Experiment 1, participants displayed significantly higher accuracy for identifying sequential violations that occurred between elements within the same sensory modality (e.g., tone-tone or shape-shape) than they did identifying violations at crossmodal boundaries (e.g., tone-shape). Likewise, participants in Experiment 2 showed significantly better performance identifying violations between elements in the same perceptual category (e.g., tone-tone, word-word, shape-shape, or colorcolor) than they did identifying violations at category boundaries within the same sensory modality (e.g., tone-word or colorshape). It appears that statistical learning is biased to operate first within a particular perceptual category, before integrating items across categories or across modalities.

The test sequences were constructed such that some items contained within-domain violations of the grammar whereas other items contained cross-domain violations. These violations were all adjacent dependencies. However, the grammar also contains subtle non-adjacent regularities that participants possibly could have learned. The statistical strength of these nonadjacent dependencies, however, is relatively weak compared to the strength of the adjacent-item dependencies, with transitional probabilities being 0.33 for the former and 0.5 for the latter (that is, the grammar stipulates two adjacentitem links for each stimulus and three non-adjacent item links for each stimulus). Because Gómez (2002) demonstrated that the learning of non-adjacent dependencies occurs only when the adjacent-item statistics are unreliable, which was not the case in the present study, it is more likely that participants' performance was based primarily on the learning of adjacent-item dependencies. Regardless, even if some amount of non-adjacent item statistics were learned, it does not much change the overall finding of this study, which is that crossdomain sequential dependencies appear to be difficult to learn.

These findings furthermore provide evidence that is not entirely consistent with a purely domain-general view of statistical learning (Altmann et al., 1995; Manza and Reber, 1997; Kirkham et al., 2002), in which all dependencies would be expected to be treated the same and learned at comparable levels. Instead, the present findings are consistent with previous research suggesting the presence of modality constraints and perceptual grouping principles affecting statistical learning (Creel et al., 2004; Conway and Christiansen, 2005, 2006; Gebhart et al., 2009; Emberson et al., 2011). Although learning across domains may operate via similar computational principles, it 
has been argued that there may exists a distributed network of modality-constrained learning mechanisms (Conway and Christiansen, 2005; Frost et al., 2015). Under this view, learning associations between stimuli taking on the same sensory and perceptual characteristics takes precedence over the learning of associations across perceptual categories and modalities. Furthermore, previous evidence suggests that each sensory modality computes statistical associations for its particular input type only, with patterns learned through one sensory modality or perceptual category staying representationally bound to that particular domain (Conway and Christiansen, 2006). However, likely due to differences in attentional or memory requirements, cross-modal statistical learning appears to be possible when the cross-modal contingencies occur together in time, rather than across a temporal sequence as was the case in the present study (Seitz et al., 2007; Sell and Kaschak, 2009; Cunillera et al., 2010; Thiessen, 2010; Mitchel and Weiss, 2011; Mitchel et al., 2014).

This type of hierarchical arrangement for statistical learning perhaps is not surprising given what we know about basic brain organization. In general, hierarchically lower level (i.e., upstream) brain areas mediate the processing of specific stimulus properties (e.g., color, motion, pitch, etc.) whereas at increasingly hierarchically higher (i.e., downstream) brain areas, more abstract and multimodal properties are integrated (e.g., speech, complex visual objects, etc.). Although certainly perception is not entirely modular, with downstream "multimodal" regions able to influence upstream areas through feedback connections (e.g., Driver and Spence, 2000), it is clear that both segregation (at upstream levels) and integration (at downstream levels) are foundational aspects of brain organization and processing (Friston et al., 1995). From a neurobiological standpoint, it is likely that statistical-sequential learning recapitulates these general principles of segregation and integration in the brain.

Although the present study found no evidence for multimodal integration of cross-modal sequential dependencies, this does not mean that it cannot occur under different experimental conditions, for example with a longer learning phase duration or manipulations to promote attention to the cross-modal dependencies. In fact, a prominent theory of sequence learning, based upon neuroimaging and behavioral findings using the serial reaction time task, posits the existence of two partially dissociable neurocognitive learning mechanisms: an implicit unidimensional system that operates over inputs within the same perceptual modality, and a multidimensional system that operates over inputs across perceptual modalities or categories (Keele et al., 2003). Importantly, the latter system appears to require attentional resources to learn the crossmodal or cross-categorical associations (see Daltrozzo and Conway, 2014, for a similar two-system view of statistical learning). Applying such a dual-system perspective to the present findings would seem to indicate that only the implicit unidimensional system was active during this task, not the multidimensional system, presumably due to a lack of attentional focus on the cross-domain dependencies. Regardless of whether one adopts the dual-system view, it appears that even if multimodal sequential integration is possible, it is not the initial gateway to learning under standard incidental learning conditions as used in the present study. Instead, input modalities during statistical learning appear to be initially percept specific, and perhaps only become integrated at subsequent levels of processing when additional cognitive resources are deployed.

Finally, it could be argued that the manner in which multisensory statistical learning was tested, with cross-modal dependencies occurring across a temporal sequence, is not ecologically realistic of what humans or other complex organisms typically encounter in the world. To this point, we offer two considerations. First, in the present study we aimed to create a learning situation that would probe the limits of multisensory statistical learning across a temporal sequence. Even if the patterns presented to participants are not ecologically realistic, the findings provide insight about possible limitations constraining cross-domain statistical learning and provide hints to the underlying architecture of the learning mechanisms themselves. Second, the issue of ecological validity implies a certain chain of reasoning: that humans have difficulty learning cross-domain sequential patterns because they are not exposed to such patterns in the world. On the other hand, the direction of causality could in fact go in the other direction: perhaps the reason why we observe minimal cross-domain sequential dependencies in our environments is precisely because of the limitations inherent in our learning faculties. For instance, it is conceivable that natural language could have evolved to capitalize on cross-domain sequential dependencies (e.g., it is logically possible that sentences could be composed not just of sequences of auditory-vocal units, but sequences of spoken words interleaved with hand and arm gestures). The lack of such cross-domain sequential dependencies in human communication could be due to the inability of humans to effectively learn such sequential patterns, consistent with the view that natural language has evolved to adapt to the processing constraints and limitations of the human brain (Christiansen and Chater, 2008).

In sum, the results of these two experiments show that when statistical-sequential input is composed of elements from two different perceptual categories or sensory modalities, participants can detect violations that occur between elements within a single domain, but not violations that occur between domains. These findings stand in contrast to previous demonstrations of cross-modal statistical learning and provide new insights about the difficulties facing learners exposed to complex multisensory environments.

\section{AUTHOR CONTRIBUTIONS}

AW, CC both contributed to the conception and design of this work and interpretation of data; the drafting and revising of the manuscript; and final approval of the version to be published. AW furthermore carried out the acquisition and analysis of the data. 


\section{ACKNOWLEDGMENTS}

This project was supported by the following grant from the National Institute on Deafness and Other Communication Disorders: R01DC012037. The sponsor had no role in any of the following aspects of this study: the study design; collection, analysis, or interpretation of data; writing of the report; decision to submit the paper for publication. We wish to thank Adam Abegg for his programming expertise, as well as Caroline

\section{REFERENCES}

Abla, D., and Okanoya, K. (2008). Statistical segmentation of tone sequences activates the left inferior frontal cortex: a near-infrared spectroscopy study. Neuropsychologia 46, 2787-2795. doi: 10.1016/j.neuropsychologia.2008.05.012

Altmann, G. T. M., Dienes, Z., and Goode, A. (1995). Modality independence of implicitly learned grammatical knowledge. J. Exp. Psychol. Learn. Mem. Cogn. 21, 899-912.

Arciuli, J., and Simpson, I. C. (2012). Statistical learning is related to reading ability in children and adults. Cogn. Sci. 36, 286-304. doi: 10.1111/j.15516709.2011.01200.x

Chang, G. Y., and Knowlton, B. J. (2004). Visual feature learning in artificial grammar classification. J. Exp. Psychol. Learn. Mem. Cogn. 30, 714-722.

Christiansen, M. H., and Chater, N. (2008). Language as shaped by the brain. Behav. Brain Sci. 31, 489-558. doi: 10.1017/S0140525X08004998

Conway, C. M., Bauernschmidt, A., Huang, S. S., and Pisoni, D. B. (2010). Implicit statistical learning in language processing: word predictability is the key. Cognition 114, 356-371. doi: 10.1016/j.cognition.2009.10.009

Conway, C. M., and Christiansen, M. H. (2005). Modality-constrained statistical learning of tactile, visual, and auditory sequences. J. Exp. Psychol. Learn. Mem. Cogn. 31, 24-39.

Conway, C. M., and Christiansen, M. H. (2006). Statistical learning within and between modalities: pitting abstract against stimulus-specific representations. Psychol. Sci. 17, 905-912. doi: 10.1111/j.1467-9280.2006.01801.x

Conway, C. M., Goldstone, R. L., and Christiansen, M. H. (2007). "Spatial constraints on visual statistical learning of multi-element scenes," in Proceedings of the 29th Annual Meeting of the Cognitive Science Society, eds D. S. McNamara and J. G. Trafton (Austin, TX: Cognitive Science Society), 185-190.

Conway, C. M., and Pisoni, D. B. (2008). Neurocognitive basis of implicit learning of sequential structure and its relation to language processing. Ann. N. Y. Acad. Sci. 1145, 113-131. doi: 10.1196/annals.1416.009

Creel, S. C., Newport, E. L., and Aslin, R. N. (2004). Distant melodies: statistical learning of nonadjacent dependencies in tone sequences. J. Exp. Psychol. Learn. Mem. Cogn. 30, 1119-1130.

Cunillera, T., Càmara, E., Laine, M., and Rodríguez-Fornells, A. (2010). Speech segmentation is facilitated by visual cues. Q. J. Exp. Psychol. 64, 1021-1040.

Daltrozzo, J., and Conway, C. M. (2014). Neurocognitive mechanisms of statisticalsequential learning: what do event-related potentials tell us? Front. Hum. Neurosci. 8:437. doi: 10.3389/fnhum.2014.00437

Driver, J., and Spence, C. (2000). Multisensory perception: beyond modularity and convergence. Curr. Biol. 10, R731-R735. doi: 10.1016/S0960-9822(00)00740-5

Emberson, L. L., Conway, C. M., and Christiansen, M. H. (2011). Timing is everything: changes in presentation rate have opposite effects on auditory and visual implicit statistical learning. Q. J. Exp. Psychol. 64, 1021-1040. doi: $10.1080 / 17470218.2010 .538972$

Fiser, J., and Aslin, R. (2001). Unsupervised statistical learning of higher-order spatial structures from visual scenes. Psychol. Sci. 12, 499-504.

Frensch, P. A., and Runger, D. (2003). Implicit learning. Curr. Dir. Psychol. Sci. 12, 13-18. doi: 10.1111/1467-8721.01213

Friston, K. J., Tononi, G., Sporns, O., and Edelman, G. M. (1995). Characterizing the complexity of neuronal interactions. Hum. Brain Mapp. 3, 302-314. doi: 10.1002/hbm.460030405

Frost, R., Armstrong, B. C., Siegelman, N., and Christiansen, M. H. (2015). Domain generality versus domain specificity: the paradox of statistical learning. Trends Cogn. Sci. 19, 117-125. doi: 10.1016/j.tics.2014.12.010
Hoyniak, Pooja Parupalli, Katherine Smith, and Ryan Town for their help with this project.

\section{SUPPLEMENTARY MATERIAL}

The Supplementary Material for this article can be found online at: http://journal.frontiersin.org/article/10.3389/fpsyg. 2016.00250

Gebhart, A. L., Newport, E. L., and Aslin, R. N. (2009). Statistical learning of adjacent and non-adjacent dependencies among non-linguistic sounds. Psychon. Bull. Rev. 16, 486-490. doi: 10.3758/PBR.16.3.486

Gómez, R. L. (2002). Variability and detection of invariant structure. Psychol. Sci. 13, 431-436. doi: 10.1111/1467-9280.00476

Jamieson, R. K., and Mewhort, D. J. K. (2005). The influence of grammatical, local, and organizational redundancy on implicit learning: an analysis using information theory. J. Exp. Psychol. Learn. Mem. Cogn. 31, 9-23.

Joseph, R. M., Steele, S. D., Meyer, E., and Tager-Flusberg, H. (2005). Selfordered pointing in children with autism: failure to use verbal mediation in the service of working memory. Neuropsychologia 43, 1400-1411. doi: 10.1016/j.neuropsychologia.2005.01.010

Karuza, E. A., Newport, E. L., Aslin, R. A., Starling, S. J., Tivarus, M. E., and Bavelier, D. (2013). The neural correlates of statistical learning in a word segmentation task: an fMRI study. Brain Lang. 127, 46-54. doi: 10.1016/j.bandl.2012.11.007

Keele, S. W., Ivry, R., Mayr, U., Hazeltine, E., and Heuer, H. (2003). The cognitive and neural architecture of sequence representation. Psychol. Rev. 110, 316-339. doi: 10.1037/0033-295X.110.2.316

Kidd, E. (2012). Implicit statistical learning is directly associated with the acquisition of syntax. Dev. Psychol. 48, 171-184. doi: 10.1037/a00 25405

Kirkham, N. Z., Slemmer, J. A., and Johnson, S. P. (2002). Visual statistical learning in infancy: evidence for a domain general learning mechanism. Cognition 83, B25-B42. doi: 10.1016/S0010-0277(02)00004-5

Lieberman, M. D., Chang, G. Y., Chiao, J., Bookheimer, S. Y., and Knowlton, B. J. (2004). An event-related fMRI study of artificial grammar learning in a balanced chunk strength design. J. Cogn. Neurosci. 16, 427-438. doi: $10.1162 / 089892904322926764$

Manza, L., and Reber, A. S. (1997). "Representing artificial grammars: transfer across stimulus forms and modalities," in How Implicit is Implicit Learning?, ed. D. C. Berry (New York, NY: Oxford University Press), 73-106.

Misyak, J. B., and Christiansen, M. H. (2012). Statistical learning and language: an individual differences study. Lang. Learn. 62, 302-331. doi: 10.1111/j.14769922.2010.00626.x

Misyak, J. B., Christiansen, M. H., and Tomblin, J. B. (2010). Sequential expectations: the role of prediction-based learning in language. Top. Cogn. Sci. 2, 138-153. doi: 10.1111/j.1756-8765.2009.01072.x

Mitchel, A. D., Christiansen, M. H., and Weiss, D. J. (2014). Multimodal integration in statistical learning: evidence from the McGurk illusion. Front. Psychol. 5:407. doi: 10.3389/fpsyg.2014.00407

Mitchel, A. D., and Weiss, D. J. (2010). What's in a face? Visual contributions to speech segmentation. Lang. Cogn. Process. 25, 456-482. doi: $10.1080 / 01690960903209888$

Mitchel, A. D., and Weiss, D. J. (2011). Learning across senses: cross-modal effects in multisensory statistical learning. J. Exp. Psychol. Learn. Mem. Cogn. 37, 1081-1091. doi: 10.1037/a0023700

Nemeth, D., Janacsek, K., Csifcsak, G., Szvoboda, G., Howard, J. H., and Howard, D. V. (2011). Interference between sentence processing and probabilistic implicit sequence learning. PLoS ONE 6:e17577. doi: 10.1371/journal.pone.0017577

Newport, E. L., and Aslin, R. N. (2004). Learning at a distance I. Statistical learning of non-adjacent dependencies. Cogn. Psychol. 48, 127-162. doi: 10.1016/S00100285(03)00128-2 
Opitz, B., and Friederici, A. D. (2004). Brain correlates of language learning: the neuronal dissociation of rule-based versus similarity-based learning. J. Neurosci. 24, 8436-8440. doi: 10.1523/JNEUROSCI.2220-04.2004

Perruchet, P., and Pacton, P. (2006). Implicit learning and statistical learning: one phenomenon, two approaches. Trends Cogn. Sci. 10, 233-238. doi: 10.1016/j.tics.2006.03.006

Petersson, K. M., Forkstam, C., and Ingvar, M. (2004). Artificial syntactic violations activate Broca's region. Cogn. Sci. 28, 383-407. doi: 10.1016/j.cogsci.2003.12.003

Reber, A. S. (1967). Implicit learning of artificial grammars. J. Verbal Learn. Verbal Behav. 6, 855-863. doi: 10.1016/S0022-5371(67)80149-X

Reber, P. J., Stark, C. E. L., and Squire, L. R. (1998). Cortical areas supporting category learning identified using functional MRI. Proc. Natl. Acad. Sci. U.S.A. 95, 747-750. doi: 10.1073/pnas.95.2.747

Robinson, C. W., and Sloutsky, V. M. (2007). "Visual statistical learning: getting some help from the auditory modality," in Proceedings of the 29th Annual Cognitive Science Society (Austin, TX: Cognitive Science Society), 611-616.

Rosenblum, L. D. (2008). Speech perception as a multimodal phenomenon. Curr. Dir. Psychol. Sci. 17, 405-409. doi: 10.1111/j.1467-8721.2008. 00615.x

Saffran, J. R., Aslin, R. N., and Newport, E. N. (1996). Statistical learning by 8month old infants. Science 274, 1926-1928. doi: 10.1126/science.274.5294.1926

Schapiro, A. C., Gregory, E., Landau, B., McCloskey, M., and Turk-Browne, N. B. (2014). The necessity of the medial temporal lobe for statistical learning. J. Cogn. Neurosci. 26, 1736-1747. doi: 10.1162/jocn_a_00578
Seger, C. A. (1994). Implicit learning. Psychol. Bull. 115, 163-196. doi: 10.1037/0033-2909.115.2.163

Seitz, A. R., Kim, R., Wassenhoven, V., and Shams, L. (2007). Simultaneous and independent acquisition of multisensory and unisensory associations. Perception 36, 1445-1453. doi: 10.1068/p5843

Sell, A. J., and Kaschak, M. P. (2009). Does visual speech information affect word segmentation? Mem. Cogn. 37, 889-894. doi: 10.3758/MC.37.6.889

Thiessen, E. D. (2010). Effects of visual information on adults' and infants' auditory statistical learning. Cogn. Sci. 34, 1093-1106. doi: 10.1111/j.15516709.2010.01118.x

Turk-Browne, N. B., Scholl, B. J., Chun, M. M., and Johnson, M. K. (2009). Neural evidence of statistical learning: efficient detection of visual regularities without awareness. J. Cogn. Neurosci. 21, 1934-1945. doi: 10.1162/jocn.2009.21131

Conflict of Interest Statement: The authors declare that the research was conducted in the absence of any commercial or financial relationships that could be construed as a potential conflict of interest.

Copyright (c) 2016 Walk and Conway. This is an open-access article distributed under the terms of the Creative Commons Attribution License (CC BY). The use, distribution or reproduction in other forums is permitted, provided the original author(s) or licensor are credited and that the original publication in this journal is cited, in accordance with accepted academic practice. No use, distribution or reproduction is permitted which does not comply with these terms. 